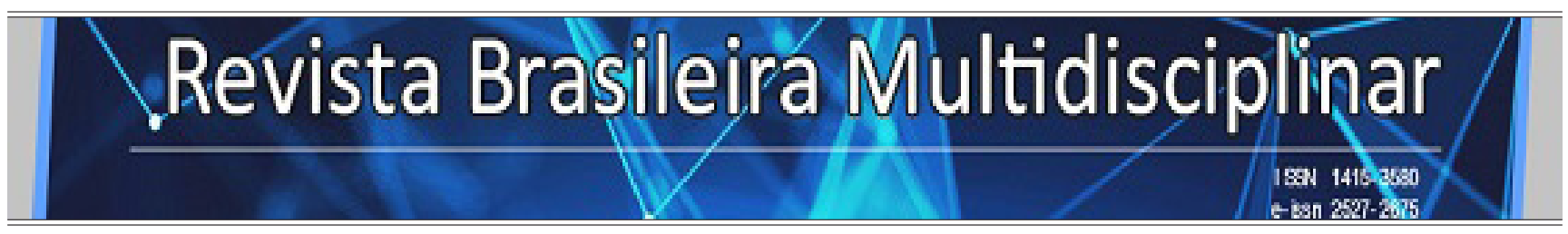

http://revistarebram.com/index.php/revistauniara

\title{
CARACTERÍSTICAS CINEMÁtICAS DA MARCHA EM INDIVÍDUOS COM INSTABILIDADE ANTERIOR DO JOELHO
}

Eduardo Moreno Judice de Mattos Farina*; Lucas Pereira Sarmento*; Luiz Augusto Mariz Gomes*; Fabrício Nascimento Almeida*; Saulo Gomes de Oliveira*

* Hospital Santa Casa de Misericórdia de Vitória.

*Autor para correspondência e-mail: eduardofarina61@gmail.com

\section{Palavras-chave}

Ligamento Cruzado Anterior Joelho

Marcha

Cinemática

\section{KEYWORDS}

Anterior Cruciate Ligament Knee

Gait

Kinematics

\begin{abstract}
RESUMO
Verificar alterações na análise dos padrões computadorizados da marcha em pacientes com instabilidade anterior do joelho, comparando com pacientes sem instabilidade articular. Vinte e sete pacientes diagnosticados com lesão do Ligamento Cruzado Anterior (LCA) do joelho (Grupo Instabilidade) e 20 indivíduos assintomáticos (Grupo Controle) foram submetidos à avaliação cinemática tridimensional da marcha. Foram comparadas a angulação média do quadril e joelho em quatro subfases, bem como as médias das angulações do quadril, joelho e tornozelo em toda a fase de apoio e balanço. Observadas maiores médias de abdução do quadril, flexão e varo do joelho na subfase de contato inicial, maiores médias de flexão e varo do joelho no apoio médio, maiores médias de flexão do quadril e varo do joelho no balanço médio no Grupo Instabilidade. Em média, em toda a fase de apoio, maior flexão do quadril, maior varo e menor rotação interna do joelho, e maiores dorsiflexão e eversão do tornozelo no Grupo Instabilidade. Por sua vez, em toda a fase de balanço, maiores médias de flexão do quadril, maiores flexão, varo e rotação externa do joelho, e menores de flexão plantar e varo do tornozelo, no Grupo Instabilidade. Foram encontradas, no Grupo Instabilidade, alterações nos padrões computadorizados da marcha nos três planos espaciais, com a presença de um padrão de anulação do quadríceps, aumento do momento adutor e da rotação externa do joelho, que podem contribuir para a degeneração articular a médio e longo prazo.
\end{abstract}

\begin{abstract}
KINETIC CHARACTERISTICS OF THE GAIT IN INDIVIDUALS WITH ANTERIOR KNEE INSTABILITY This study aims to verify alterations in the analysis of the computerized gait patterns in patients with anterior knee instability comparing with patients without joint instability. Twenty-seven patients diagnosed with anterior cruciate ligament injury (ACL) of the knee (Instability Group) and 20 asymptomatic individuals (Control Group) underwent three-dimensional kinematics evaluation of gait. The mean hip and knee angulation were compared in four subphases, as well as the means of hip, knee and ankle angulations throughout the stance and swing phases. We observed higher mean hip abduction, flexion and knee varus in the initial contact subphase, higher flexion mean and knee varus in the midstance, higher mean hip flexion and knee varus in the mean swing in the Instability Group. On average, throughout the stance phase, greater hip flexion, greater varus and lower internal rotation of the knee, and greater dorsiflexion and ankle eversion in the Instability Group. On the other hand, throughout the swing phase, greater means of hip flexion, greater flexion, varus and external rotation of the knee, and smaller plantar flexion and varus of the ankle, in the Instability Group. In the Instability Group changes were found in the computerized gait patterns in the three spatial planes, with the presence of a quadriceps avoidance pattern, increased adductive moment and external rotation of the knee that may contribute to medium and long term joint degeneration.
\end{abstract}




\section{INTRODUÇÃo}

A lesão do ligamento cruzado anterior (LCA) é uma das mais frequentes lesões que acometem a articulação do joelho, mais frequente em pacientes jovens e esportistas (LOHMANDER, 2007). Esse ligamento desempenha um importante papel na cinemática articular tibiofemoral, não apenas pela limitação da translação tibial anterior, mas também pela estabilização da rotação axial do joelho, mesmo durante atividades rotineiras como a caminhada (LIU X., 2020).

As rupturas do LCA alteram os padrões de movimento de todo o membro inferior influenciando no controle de ativação muscular, como a inibição do quadríceps, na tentativa de evitar aumento da translação anterior, resultando em alterações compensatórias para a dor e a instabilidade do joelho (HURD, SNYDER-MACKLER, 2007; SHELBURNE, TORRY, PANDY, 2005; BERCHUCK, 1990; SHARIFI, SHIRAZI-ADL, MAROUANE, 2020). Porém, após a ruptura ligamentar, tais adaptações não conseguem proteger o desenvolvimento de condições patológicas secundárias, como lesões meniscais e condrais, bem como a osteoartrose (DANIEL, STONE, DOBSON, 1994; PAPAGEORGIOU, GIL, KANAMORI, 2001; SLATER, 2017 ).

A progressão para osteoartrose em pacientes com insuficiência do LCA ocorre principalmente no compartimento medial, devido ao aumento do momento adutor do joelho identificado na análise da marcha no plano coronal. Estudos demonstram que a cinemática articular modificada do joelho está associada a um aumento de $44 \%$ de perda de cartilagem no compartimento medial em comparação a um joelho com LCA preservado (BUTLER, 2011; HURWITZ, 1998; MIYAZAKI, 2002; ANDRIACCHI, 2006). Além dos desvios da marcha no plano coronal, aumentos significativos na rotação interna da tíbia durante a fase de apoio da marcha também foram relatados (ANDRIACCHI, DYRBY, 2005; GEORGOULIS, 2003).

Apesar de ser bem conhecida a história natural da ruptura do LCA, ainda existem dúvidas das mudanças cinemáticas na marcha nesses indivíduos. Evidências limitadas e escassez de trabalhos, associando as alterações cinemáticas e os padrões de caminhada com a instabilidade anterior articular e o desenvolvimento de osteoartrose, foram apresentadas recentemente por Ismail (2016) em uma revisão sistemática, tornando-se útil especificá-las detalhadamente, além de determinar os mecanismos compensatórios no tornozelo e quadril.

A ruptura do LCA é uma patologia frequente na população economicamente ativa, podendo progredir para osteoartorse e gerar impactos econômicos e na saúde pública. O objetivo desse estudo é verificar se existem alterações na análise dos padrões computadorizados da marcha em pacientes com ruptura do LCA, comparando com os padrões de pacientes sem instabilidade articular, para averiguar se o tratamento cirúrgico dessa patologia deve ser considerado mesmo em pacientes sem sintomatologia articular.

\section{MATERIAIS E MÉTOdos}

Após aprovação do Comitê de Ética (número de aprovação: 1.538.444), realizou-se um estudo transversal e observacional com 27 pacientes com sintomas de instabilidade anterior do joelho atendidos no Ambulatório do Joelho do Hospital da Santa Casa de Misericórdia de Vitória, com diagnóstico clínico e por Ressonância Magnética de ruptura do LCA (Grupo Instabilidade), comparados com 20 pacientes com ambos os joelhos assintomáticos e sem instabilidade ligamentar (Grupo Controle), com idade entre $18 \mathrm{e}$ 45 anos. A instabilidade articular foi definida através de manobras do exame físico com Sinal da Gaveta Anterior, Lachman e Pivot Shift, realizadas sempre por 2 médicos ortopedistas especialistas em joelho. Foram excluídos os pacientes que apresentavam sintomas articulares no membro inferior contralateral à lesão do LCA, rupturas multiligamentares incluindo o LCA ou outras condições no joelho pesquisado que impediam ou limitavam a adequada realização dos testes. Pacientes com lesão do LCA em ambos os joelhos foram excluídos da análise, visto que alteraria a homogeneidade da amostra de pacientes com 
lesão em somente um joelho.

Inicialmente, realizou-se o cálculo da velocidade média individual a partir do tempo necessário para caminhar uma distância de 10 metros em terreno liso, de horizontalização neutra, de maneira confortável e rotineira de cada paciente. Em seguida, foi realizada uma análise computadorizada tridimensional da marcha em uma esteira na posição horizontal a $0^{\circ}$ de angulação, na velocidade média individual, previamente calculada durante aproximadamente 5 a 10 minutos, com medidas das variáveis angulares dos quadris, joelhos e tornozelos, pelo sistema computadorizado Noraxon MR3 ${ }^{\circledast}$ (v3.8) por via de sensores aderentes à pele em ambos os membros inferiores do paciente, com fitas de velcro localizados nos pés $\left(1^{\text {a }}\right.$ cabeça metatarsal), tíbia proximal (tuberosidade anterior da tíbia), fêmur distal ( $03 \mathrm{~cm}$ acima da base da patela) e cintura pélvica (01 cm acima das asas do ilíaco) (Figura1).

Figura 1- Vista posterior, anterior e lateral do posicionamento dos sensores da avaliação tridimensional da marcha.

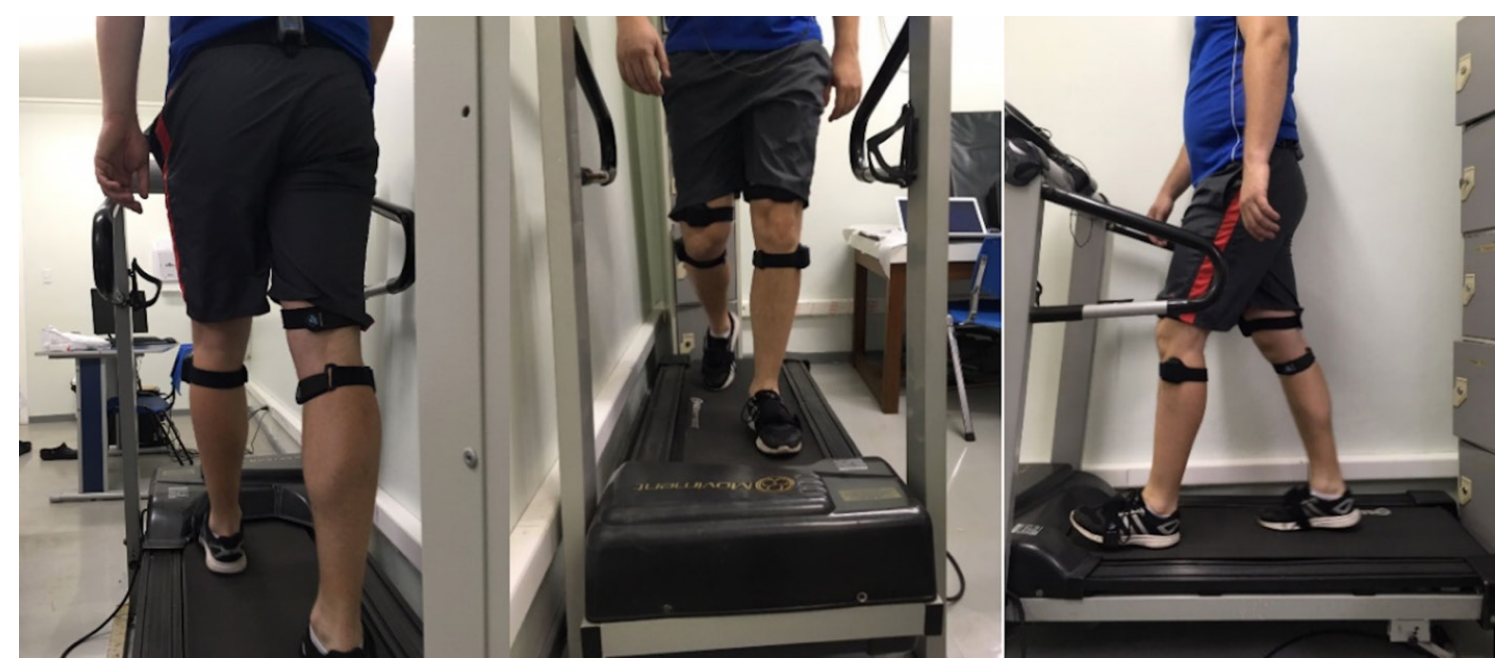

Fonte: Foto do autor, 2017.

Os dados do quadril e joelho foram representados em um Avatar (Figura 2) criado pelo software, que possibilitou o registro das angulações articulares dos membros inferiores durante o primeiro ciclo da marcha, imediatamente após o primeiro minuto de caminhada, considerado como período de adaptação ao teste.

Os dados foram posteriormente coletados em ambos os grupos pelo congelamento da imagem do avatar nos planos espaciais coronal e sagital, considerando três eventos ocorridos em três subfases da fase de apoio: Contato Inicial (apoio do calcâneo), Apoio Médio (sobreposição dos dois joelhos) e Apoio Terminal (desprendimento do calcâneo); e um evento ocorrido na subfase de Balanço Médio da fase de balanço (sobreposição dos dois joelhos). As imagens foram transpostas para o programa CorelDRAWGraphics Suite 2017® para cálculo das angulações do quadril e joelho, utilizando o centro da cabeça femoral e o côndilo lateral do fêmur como referência para a articulação do quadril e as linhas médio-diafisárias do fêmur e da tíbia parao joelho. O cálculo das angulações do tornozelo foram dificultadas pela impossibilidade de padronização de uma referência óssea confiável no tornozelo e pé. As avaliações no plano transversal também foram impossibilitadas pela sobreposição do fêmur e tíbia com as demais referências ósseas, sendo optado por não realizar essas aferições. Os dados fornecidos pelo software com as médias e desvio padrão das articulações do quadril, joelho e tornozelo nos planos sagital, coronal e transversal de toda a fase de apoio e balanço, sem divisão em subfases, também foram coletados. 
Figura 2- Vista coronal e lateral da representação do avatar utilizado para cálculo das variáveis angulares do quadril e joelho. A cor vermelha representa o movimento das articulações do membro inferior esquerdo, e a verde, do membro inferior direito.

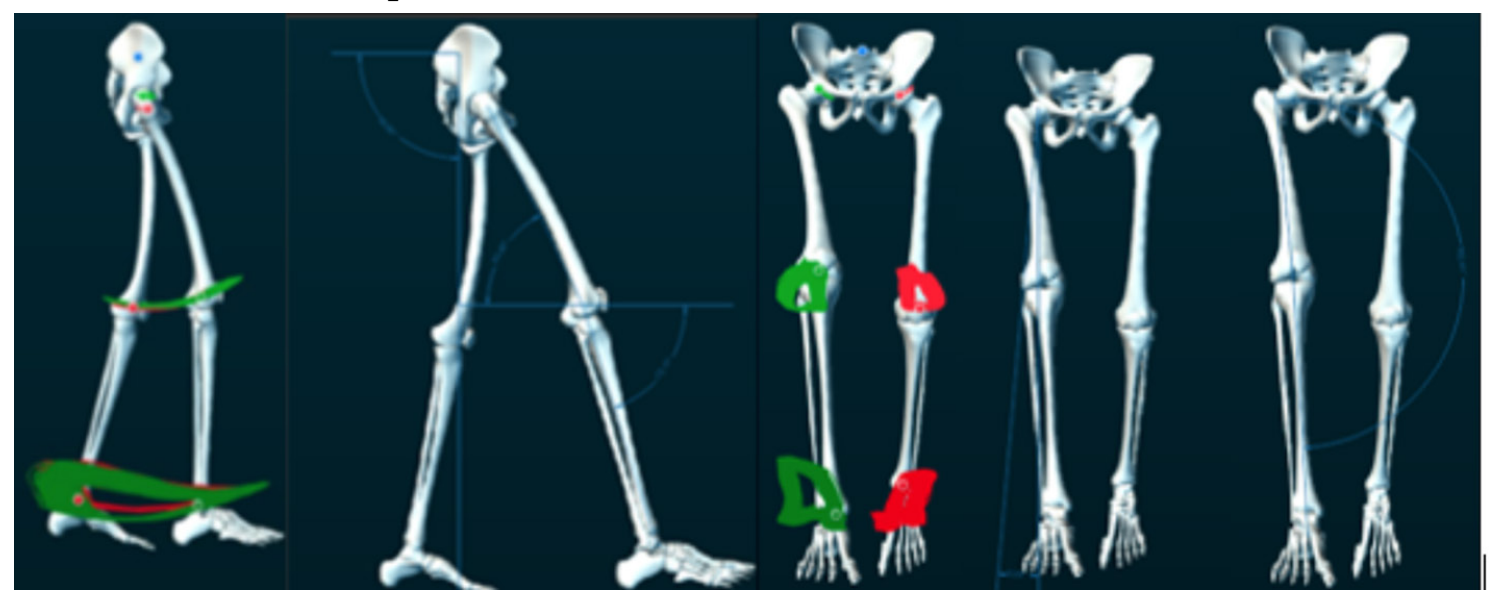

Fonte: Foto do autor por meio do Software Noraxon MR3 ${ }^{\oplus}$

Considerou-se a linha média vertical como valor $0^{\circ}$ (zero) para os planos sagital e coronal e a linha média horizontal como $0^{\circ}$ (zero) para o plano transversal, além de positivos $(+)$ os valores angulares de abdução, flexão e rotação externa, e negativos (-) os valores de adução, extensão e rotação interna nos planos coronal, sagital e transversal respectivamente.

As informações dos valores angulares do quadril e joelho, nas quatro subfases calculadas pelo software Noraxon $\mathrm{MR}^{\circledR}$ nos planos coronal e sagital (dados obtidos pela posição tridimensional dos sensores aderidos à pele), e as médias dos valores angulares tridimensionais de toda a fase de apoio e balanço no quadril, joelho e tornozelo do membro inferior afetado do Grupo Instabilidade determinados pelo software foram comparados com os dados de ambos os membros inferiores do Grupo Controle.

Os dados coletados manualmente nas quatro subfases da marcha apresentaram distribuição normal de probabilidade pelo teste de Kolmogorov-Smirnov a 5\% de significância, permitindo a comparação das variáveis pelo teste paramétrico t-Student. Em contrapartida, os resultados coletados pelo software de toda a fase de apoio e balanço apresentaram distribuição anormal de probabilidade, sendo optado pelo uso do teste não paramétrico de Mann-Whitney. Todos os cálculos foram realizados pelo software SPSS 20.0 , considerando estatisticamente significantes os valores $\mathrm{p}<0,05$.

\section{RESUlTADOS}

o Grupo instabilidade consistia de 24 indivíduos masculinos e 03 femininos, com média de idade 31,14 anos, Índice de Massa Corporal (IMC) médio 26,0 Kg/m² e sintomas de instabilidade entre 06 e 12 meses. O grupo controle foi representado por 11 pessoas do sexo masculino e 09 feminino, com média de idade 32,5 anos e IMC médio $22,7 \mathrm{Kg} / \mathrm{m}^{2}$.

A Quadro 1 e a Quadro 2 apresentam os resultados angulares encontrados do quadril e o valor de significância entre os grupos nos planos sagital e coronal respectivamente. A Quadro 3 e a Quadro 4 apresentam os mesmos resultados da articulação do joelho nos planos sagital e coronal, respectivamente. A Quadro 5 e a Quadro 6 exibem os resultados dos valores angulares nos três planos durante a toda a fase de apoio e balanço, respectivamente. 
Quadro 1 -Resultados observados no quadril no plano Sagital (flexo-extensão) durante as subfases da marcha.

\begin{tabular}{|c|c|c|c|c|c|}
\hline Subfase da marcha & Grupo & $\mathbf{N}$ & Médias & Desvio Padrão & Valor de $p$ \\
\hline \multirow{2}{*}{ Contato inicial } & Instabilidade & 27 & 30,21 & 6,73 & \multirow{2}{*}{0,448} \\
\hline & Controle & 20 & 28,68 & 6,82 & \\
\hline \multirow{2}{*}{ Apoio Médio } & Instabilidade & 27 & 12,26 & 4,37 & \multirow{2}{*}{0,803} \\
\hline & Controle & 20 & 11,91 & 4,98 & \\
\hline \multirow{2}{*}{ Apoio terminal } & Instabilidade & 27 & $-7,45$ & 4,72 & \multirow{2}{*}{0,707} \\
\hline & Controle & 20 & $-8,15$ & 7,82 & \\
\hline \multirow{2}{*}{ Balanço Médio } & Instabilidade & 27 & 15,63 & 4,57 & \multirow{2}{*}{0,011} \\
\hline & Controle & 20 & 11,85 & 5,19 & \\
\hline
\end{tabular}

Fonte: elaborado pelos autores.

Quadro 2 - Resultados observados no quadril no plano Coronal (abdução-adução) durante as subfases da marcha.

\begin{tabular}{|c|c|c|c|c|c|}
\hline $\begin{array}{c}\text { Subfase da } \\
\text { marcha }\end{array}$ & Grupo & $\mathbf{N}$ & Médias & Desvio Padrão & Valor de $p$ \\
\hline \multirow{2}{*}{ Contato Inicial } & Instabilidade & 27 & 11,46 & 5,49 & \multirow{2}{*}{0,023} \\
\hline & Controle & 20 & 7,45 & 6,19 & \\
\hline \multirow{2}{*}{ Apoio Médio } & Instabilidade & 27 & 6,73 & 3,66 & \multirow{2}{*}{0,254} \\
\hline & Controle & 20 & 5,47 & 3,75 & \\
\hline \multirow{2}{*}{ Apoio terminal } & Instabilidade & 27 & 6,98 & 3,70 & \multirow{2}{*}{0,206} \\
\hline & Controle & 20 & 8,33 & 3,37 & \\
\hline \multirow{2}{*}{ Balanço Médio } & Instabilidade & 27 & 13,00 & 4,45 & \multirow{2}{*}{0,602} \\
\hline & Controle & 20 & 12,26 & 5,11 & \\
\hline
\end{tabular}

Fonte: elaborado pelos autores.

Quadro 3 - Resultados observados no joelho no plano Sagital (flexo-extensão) durante as subfases da marcha.

\begin{tabular}{|c|c|c|c|c|c|}
\hline Subfase da marcha & Grupo & $\mathbf{N}$ & Médias & Desvio Padrão & Valor de $p$ \\
\hline \multirow{2}{*}{ Contato inicial } & Instabilidade & 27 & 26,81 & 11,67 & \multirow{2}{*}{0,001} \\
\hline & Controle & 20 & 15,71 & 8,19 & \\
\hline \multirow{2}{*}{ Apoio Médio } & Instabilidade & 27 & 15,85 & 6,87 & \multirow{2}{*}{0,043} \\
\hline & Controle & 20 & 11,85 & 5,95 & \\
\hline \multirow{2}{*}{ Apoio terminal } & Instabilidade & 27 & 17,14 & 10,91 & \multirow{2}{*}{0,676} \\
\hline & Controle & 20 & 18,39 & 8,74 & \\
\hline \multirow{2}{*}{ Balanço Médio } & Instabilidade & 27 & 54,11 & 9,71 & \multirow{2}{*}{0,790} \\
\hline & Controle & 20 & 53,41 & 7,80 & \\
\hline
\end{tabular}

Fonte: elaborado pelos autores. 
Quadro 4 - Resultados observados no joelho no plano Coronal (abdução-adução) durante as subfases da marcha.

\begin{tabular}{|c|c|c|c|c|c|}
\hline Subfase da marcha & Grupo & $\mathbf{N}$ & Médias & Desvio Padrão & Valor de p \\
\hline \multirow[t]{2}{*}{ Contato inicial } & Instabilidade & 27 & $-3,96$ & 6,58 & \multirow[t]{2}{*}{0,002} \\
\hline & Controle & 20 & 1,45 & 4,21 & \\
\hline \multirow{2}{*}{ Apoio Médio } & Instabilidade & 27 & $-2,63$ & 4,15 & \multirow{2}{*}{0,030} \\
\hline & Controle & 20 & $-0,07$ & 3,50 & \\
\hline \multirow[t]{2}{*}{ Apoio terminal } & Instabilidade & 27 & $-3,34$ & 8,73 & \multirow[t]{2}{*}{0,348} \\
\hline & Controle & 20 & $-1,10$ & 6,82 & \\
\hline \multirow{2}{*}{ Balanço Médio } & Instabilidade & 27 & $-12,86$ & 8,59 & \multirow{2}{*}{0,031} \\
\hline & Controle & 20 & $-5,36$ & 14,41 & \\
\hline
\end{tabular}

Fonte: elaborado pelos autores. 
Quadro 5 - Valores angulares observados nos três planos durante a toda a fase de apoio.

\begin{tabular}{|c|c|c|c|c|c|}
\hline \multirow[b]{3}{*}{ QUADRIL } & \multicolumn{4}{|c|}{ GRUPO } & \multirow{3}{*}{ Valor de $\mathrm{p}$} \\
\hline & \multicolumn{2}{|c|}{ Instabilidade $(\mathrm{N}=27)$} & \multicolumn{2}{|c|}{ Controle $(\mathrm{N}=20)$} & \\
\hline & Média & $\begin{array}{l}\text { Desvio } \\
\text { Padrão }\end{array}$ & Média & $\begin{array}{l}\text { Desvio } \\
\text { Padrão }\end{array}$ & \\
\hline $\begin{array}{c}\text { Sagital } \\
\text { (Flexo-extensão) }\end{array}$ & 17,25 & 8,55 & 8,18 & 6,82 & 0,001 \\
\hline $\begin{array}{c}\text { Coronal } \\
\text { (Abdução-Adução) }\end{array}$ & $-4,38$ & 4,24 & $-4,37$ & 2,84 & 0,745 \\
\hline $\begin{array}{c}\text { Transversal } \\
\text { (Rot. Interna-Externa) }\end{array}$ & 1,03 & 9,46 & 0,25 & 2,34 & 0,179 \\
\hline \multicolumn{6}{|l|}{ JOELHO } \\
\hline $\begin{array}{c}\text { Sagital } \\
\text { (Flexão-Extensão) }\end{array}$ & 13,70 & 7,61 & 12,29 & 5,82 & 0,324 \\
\hline $\begin{array}{c}\text { Coronal } \\
\text { (Abdução/Valgo-Adução/ } \\
\text { Varo) }\end{array}$ & $-1,84$ & 3,54 & 0,81 & 2,53 & 0,013 \\
\hline $\begin{array}{c}\text { Transversal } \\
\text { (Rot. Interna-Externa) }\end{array}$ & 1,00 & 4,41 & 2,59 & 3,19 & 0,005 \\
\hline \multicolumn{6}{|l|}{ TORNOZELO } \\
\hline $\begin{array}{c}\text { Sagital } \\
\text { (Flexão/Dorsiflexão- } \\
\text { Extensão/Flexão Plantar) }\end{array}$ & 2,54 & 3,18 & $-4,09$ & 3,53 & 0,001 \\
\hline $\begin{array}{c}\text { Coronal } \\
\text { (Abdução/Valgo-Adução/ } \\
\text { Varo) }\end{array}$ & $-1,46$ & 4,35 & $-2,76$ & 4,96 & 0,230 \\
\hline $\begin{array}{c}\text { Transversal } \\
\text { (Inversão/Rot. Interna- } \\
\text { Eversão/Rot. Externa) }\end{array}$ & $-3,77$ & 3,73 & $-1,43$ & 3,99 & 0,017 \\
\hline
\end{tabular}

Fonte: elaborado pelos autores. 
Quadro 6 - Valores angulares observados nos três planos durante a toda a fase de balanço.

\begin{tabular}{|c|c|c|c|c|c|}
\hline \multirow[b]{3}{*}{ QUADRIL } & \multicolumn{4}{|c|}{ GRUPO } & \multirow{3}{*}{ Valor de $\mathrm{p}$} \\
\hline & \multicolumn{2}{|c|}{ Instabilidade $(\mathrm{N}=27)$} & \multicolumn{2}{|c|}{ Controle $(\mathrm{N}=20)$} & \\
\hline & Média & $\begin{array}{l}\text { Desvio } \\
\text { Padrão }\end{array}$ & Média & $\begin{array}{l}\text { Desvio } \\
\text { Padrão }\end{array}$ & \\
\hline $\begin{array}{c}\text { Sagital } \\
\text { (Flexo-extensão) }\end{array}$ & 19,04 & 8,5 & 9,51 & 7,2 & 0,001 \\
\hline $\begin{array}{c}\text { Coronal } \\
\text { (Abdução-Adução) }\end{array}$ & 0,41 & 4,32 & $-0,79$ & 3,03 & 0,597 \\
\hline $\begin{array}{c}\text { Transversal } \\
\text { (Rot. Interna-Externa) }\end{array}$ & 4,02 & 8,82 & 2,19 & 2,42 & 0,542 \\
\hline JOELHO & & & & & \\
\hline $\begin{array}{c}\text { Sagital } \\
\text { (Flexão-Extensão) }\end{array}$ & 37,41 & 8,46 & 26,36 & 9,92 & 0,001 \\
\hline $\begin{array}{c}\text { Coronal } \\
\text { (Abdução/Valgo-Adução/ } \\
\text { Varo) }\end{array}$ & $-4,98$ & 6,17 & 0,26 & 4,21 & 0,004 \\
\hline $\begin{array}{c}\text { Transversal } \\
\text { (Rot. Interna-Externa) }\end{array}$ & $-0,31$ & 4,94 & 2,98 & 3,3 & 0,001 \\
\hline TORNOZELO & & & & & \\
\hline $\begin{array}{c}\text { Sagital } \\
\text { (Flexão/Dorsiflexão- } \\
\text { Extensão/Flexão Plantar) }\end{array}$ & $-2,82$ & 5,95 & $-9,34$ & 6,18 & 0,001 \\
\hline $\begin{array}{c}\text { Coronal } \\
\text { (Abdução/Valgo-Adução/ } \\
\text { Varo) }\end{array}$ & $-3,14$ & 3,66 & $-5,35$ & 4,8 & 0,048 \\
\hline
\end{tabular}

Fonte: elaborado pelos autores. 


\section{Discuss Ão}

Durante a subfase de contato inicial, as articulações dos pacientes com instabilidade anterior do joelho apresentaram maiores médias de abdução do quadril, assim como flexão e varo do joelho, quando comparadas com grupo controle ( $\mathrm{p}<0,05$, quadros 2 a 4 ), alterações não notadas ao exame físico. $\mathrm{Na}$ subfase de apoio médio, observaram-se maiores médias de flexão e varo do joelho, e no balanço médio, constataram-se maiores médias de flexão do quadril e varo do joelho no Grupo Instabilidade.

Quando analisadas as médias de toda a fase de apoio, verificou-se maior flexão do quadril, maior varo e menor rotação interna do joelho, bem como maiores dorsiflexão e eversão do tornozelo no Grupo Instabilidade. $\mathrm{Na}$ fase de balanço, maiores médias de flexão do quadril, maiores de flexão, varo e rotação externa do joelho, e menores de flexão plantar e varo do tornozelo, também no Grupo Instabilidade.

No plano coronal, a caracterização de um momento adutor do joelho, observado pelas médias do varo no Grupo Instabilidade, tanto na fase de apoio quanto na de balanço, divergiram do valgo predominante do Grupo Controle, assemelhando-se aos resultados encontrados por Gao e Zheng (2010). Em contrapartida, Zhang et al. (2003), utilizando avaliação cinemática com goniômetro 6-DOF, sugerem que os achados de maior valgo durante a caminhada evidenciados em seu estudo, seriam um mecanismo compensatório, evitando posições mais instáveis do joelho. Além disso, outros autores, empregando análises 3D, não encontraram diferenças no plano coronal em pacientes com instabilidade anterior (ALKJAER, 2003; REN, 2020; ROBERTS, 1999).

No plano sagital, alguns autores descrevem um padrão modificado da marcha em pacientes com ruptura do LCA com flexão sustentada do joelho durante a fase de apoio (WEXLER, 1998; ANDRIACCHI., 1983; BIRAC, ANDRIACCHI, BACH, 1991; HURWITZ, 1997; REN, 2020). Berchuck (1990) observaram um aumento da amplitude de flexão do joelho em pacientes com ruptura do LCA, descrito primeiramente como Padrão de Anulação do Quadríceps e defendido como um mecanismo de compensação em busca de anular ou, ao menos, diminuir a contração do quadríceps, evitando a translação anterior da tíbia proximal em relação ao fêmur distal. Chen (2009) identificaram que essa tentativa de evitar a ação do quadríceps estava associada à maior contração dos isquiotibiais, porém, seus achados de aumento da translação anteroposterior encontrados na fase de apoio sugerem que, embora o mecanismo de adaptação exista, ainda assim mostra-se insuficiente.

Os resultados encontrados identificaram um padrão semelhante, visto que foi revelado um aumento da flexão do joelho na subfase de contato inicial (apoio do calcâneo), apesar de os resultados de toda a fase de apoio não mostrarem essa diferença. Os resultados da metanálise de Ismail (2016) contrariam esses achados, pois não identificaram diferença no ângulo de flexão do joelho em diferentes fases e subfases da marcha, a despeito da heterogeneidade observada nas variáveis.

Ainda no plano sagital, os resultados sugerem uma atitude de marcha com maior média de flexão do quadril, na maior parte do ciclo da marcha, assim como o trabalho de Ismail (2016) demonstraram que os pacientes com instabilidade anterior do joelho apresentaram uma caminhada com um maior impulso angular flexor do quadril.

Devita, Hortobagyi e Barrier (1997) sugerem que uma mudança na relação comprimento-tensão nos músculos extensores do quadril de indivíduos com insuficiência do LCA, durante a fase de apoio da marcha, pode ocorrer como um resultado de um aumento da posição de flexão do quadril, possivelmente tensionando os músculos isquiotibiais e, assim, reduzindo a translação tibial anterior. Os autores sugerem que se a posição do joelho dos pacientes permanece inalterada durante a fase de apoio, uma maior posição de flexão do quadril pode tensionar os músculos isquiotibiais e possivelmente reduzir a translação tibial anterior ao longo da fase de apoio.

No plano transversal, o joelho apresentou menores médias de rotação interna, tanto na fase de apoio 
quanto na fase de balanço, assim como os achados de Zhang (2003), que sugerem que a rotação externa acontece para amenizar a tensão sobre o LCA insuficiente. De forma diferente, Georgoulis (2003) constataram rotação externa do joelho, em média, no grupo controle no início da fase de balanço, enquanto que no grupo com ruptura do LCA a rotação era, em média, interna. Além de prejudicar o mecanismo de screw home (aparafusamento) do joelho no início da flexão, a rotação interna associada à translação anterior da tíbia pode acrescentar risco de lesão meniscal secundária e alteração degenerativa na cartilagem articular.

As alterações compensatórias no tornozelo, publicadas por Ismail (2016), encontraram critérios de metanálise para comparação, apenas no plano sagital, demonstrando não haver evidências de diferença significativa no ângulo de dorsiflexão no tornozelo nos indivíduos com deficiência do LCA. Os resultados, que encontramos, demonstraram maiores médias de dorsiflexão e eversão, durante a fase de apoio, e menores médias de flexão plantar e varo do tornozelo, na fase de balanço no Grupo Instabilidade, em relação ao Grupo Controle.

Como limitações, a falta de avaliação eletromiográfica e de controle da amostra pelo acometimento de lesões condrais e meniscais, associadas, podem ter afetado a coleta e as interpretações dos resultados.

\section{Conclusão}

Os dados desse estudo sugerem que a instabilidade anterior do joelho proporciona alterações nos padrões computadorizados da marcha nos três planos espaciais. As modificações reveladas indicam a presença de um comportamento articular semelhante ao padrão de anulação do quadríceps, descrito por Berchuck (1990) e outros autores, aumento do momento adutor e da rotação externa do joelho, que podem contribuir para a degeneração articular a médio e longo prazo. Estudos cinemáticos adicionais são necessários no entendimento da associação dessas alterações biomecânicas com a evolução clínica da osteoartrose do joelho.

\section{REFERÊNCIAS}

ALKJAER, T.; SIMONSEN, E. B.; JORGENSEN, U.; DYHRE-POULSEN, P. Evaluation of the walking pattern in two types of patients with anterior cruciate ligament deficiency: copers and non-copers. Eur. J. Appl. Physiol., v. 89, n. 3, p. 301-308, 2003.

ALMEKINDERS, L. C.; PANDARINATH, R.; RAHUSEN, F. T. Knee stability following anterior cruciate ligament rupture surgery.J Bone Joint SurgAm.,v. 86, n. 5, p. 983-987, may. 2004.

ANDERSEN, H. N.; DYHRE-POULSEN, P. The anterior cruciate ligament does play a role in controlling axial rotation in the knee. Knee Surg Sports TraumatolArthrosc., v. 5, n. 3, p. 145-149, sep. 1997.

ANDRIACCHI, T. P. Functional analysis of pre- and post-knee surgery: total knee arthroplasty and ACL reconstruction. J Biomech Eng., v. 115, p. 575-581, 1983.

ANDRIACCHI, T. P.; BRIANT, P. L., BEVILL, S. L.; KOO, S. ROTATIONAL changes at the knee after ACL injury cause cartilage thinning. Clin.Orthop.Relat.Res., v. 442, p. 39-44, 2006.

ANDRIACCHI, T. P.; DYRBY, C. O. Interactions between kinematics and loading during walking 
for the normal and ACL deficient knee.J. Biomech., v. 38, n. 2, p. 293-298, 2005.

ANDRIACCHI, T.P.; ALEXANDER, E. J.; TONEY. M. K.; DYRBY, C.; SUM, J. A point cluster method for in vivo motion analysis: applied to a study of knee kinematics. J BiomechEng.,v. 120, n. 6, p. 743-749, dez. 1998.

BERCHUCK, M.; ANDRIACCHI, T. P.; BACH, B. R.; REIDER, B. Gait adaptations by patients who have a deficient anterior cruciate ligament.J Bone Joint Surg., v. 72, p. 871-877, 1990.

BIRAC, R. C.; ANDRIACCHI, T. P.; BACH, B. R. Time related changes following ACL rupture. Trans Orthop Res Soc., v. 1, p. 231, 1991.

BULGHERONI, P.; BULGHERONI, M. V.; ANDRINI, L.; GUFFANTI, P.; CASTELLI, C. Walking in anterior cruciate ligament injuries. The Knee, v. 4, n. 3, p. 159-165, set. 1997.

BUTLER, R. J.; BARRIOS, J. A.; ROYER, T.; DAVIS, I. S. Frontal-plane gait mechanics in people with medial knee osteoarthritis are different from those in people with lateral knee osteoarthritis. Phys. Ther., v. 91, n. 8, p. 1235-1243, 2011.

CHEN, C. H.; LI, J. S.; HOSSEINI, A.; GADIKOTA, H. R.; GILL, T. J.; LI, G. Anteroposterior Stability of the Knee during the Stance Phase of Gait after Anterior Cruciate Ligament Deficiency. Gait Posture, v. 35, n. 3, p. 467-471, mar. 2009.

DANIEL, D. M.; STONE, M. L.; DOBSON, B. E.; FITHIAN D. C.; ROSSMAN, D. J.; KAUFMAN, K. R. Fate of the ACL-injured patient. A prospective outcomestudy.Am J Sports Med., v. 22, p. 632-644, 1994.

DEVITA, P.; HORTOBAGYI, T.; BARRIER, J. Gait adaptations before and after anterior cruciate ligament reconstruction surgery. Med Sci Sports Exerc, v. 29, p. 853-859, 1997.

FERBER, R.; OSTERNIG, L. R.; WOOLLACOTT, M. H.; WASIELEWSKI, N. J.; LEE, J. H.Gait mechanics in chronic ACL deficiency and subsequent repair.ClinBiomech, v. 17, n. 4, p. 274-285, may. 2002.

GAO, B.; ZHENG, N. Alterations in three-dimensional joint kinematics of anterior cruciate ligament-deficient and-reconstructed knees during walking.ClinBiomech., v. 25, p. 222-229, 2010.

GEORGOUlis, A. D., PAPADONIKOLAKIS. A.; PAPAGEORGIOU, C. D.; MITSOU, A.; STERGIOU, N. Three-dimensional tibiofemoral kinematics of the anterior cruciate ligament-deficient and reconstructed knee during walking.Am. J. Sports Med., v.31, n. 1, p. 75-79, 2003. 
HURD, W. J.; SNYDER-MACKLER, L. Knee instability after acute ACL rupture affects movement patterns during the mid-stance phase of gait. J Orthop Res., v. 25, n. 10, p. 1369-1377, oct. 2007.

HURWITZ, D. E.; ANDRIACCHI, T. P.; BUSH-JOSEPH, C. A.; BACH, B. R. Func- tional adaptations in patients with ACL-deficient knees. Exerc.Sport.Sci. Rev., v. 25, p. 1-20, 1997.

HURWITZ, D. E.; SUMNER, D. R.; ANDRIACCHI, T. P.; SUGAR, D. A. Dynamic knee loads during gait predict proximal tibial bone distribution. J. Biomech., v. 31, n. 5, p. 423-430, 1998.

ISMAIL, S. A.; BUTTON, K.; SIMIC, M.; DEURSEN, R. V.; PAPPAS, E. Three-dimensional kinematic and kinetic gait deviations in individuals with chronic anterior cruciate ligament deficient knee: A systematic review and meta-analysis. Clinical Biomechanics., v. 35, p. 68-80, 2016.

LOHMANDER, L. S.; ENGLUND, P. M.; DAHL, L. L.; ROOS, E. M., 2007. The long-term consequence of anterior cruciate ligament and meniscus injuries: osteoarthritis. Am. J. Sports Med., v. 35, n. 10, p. 1756-1769, out. 2007.

MCDANIEL, W. J. JR.; DAMERON, T. B. JR. Untreated ruptures of the anterior cruciate ligament.A follow-up study.J Bone Joint Surg., v. 62, p. 696-705, 1980.

MIYAZAKI, T.; WADA, M.; KAWAHARA, H.; SATO, M.; BABA, H.; SHIMADA, S. Dynamic load at baseline can predict radiographic disease progression in medial compartment knee osteoarthritis. Ann. Rheum. Dis., v. 61, n. 7, p. 617-622, 2002.

MURAYAMA, T.; SATO, T.; WATANABE, S.; KOBAYASHI, K.; TANIFUJI, O.; MOCHIZUKI, T.; YAMAGIWA, H.; KOGA, Y.; OMORI, G.; ENDO, N. Three-dimensional in vivo dynamic motion analysis of anterior cruciate ligament-deficient knees during squatting using geometric center axis of the femur. Journal of Orthopaedic Science, v. 21, p. 159-165, 2016.

PAPAGEORGIOU, C. D.; GIL, J. E., KANAMORI, A.; FENWICK, J. A.; WOO, S. L.; FU, F. H. The biomechanical interde- pendence between the anterior cruciate ligament replacement graft and the medial meniscus.Am J Sports Med., v. 29, p. 226-231, 2001.

ROBERTS, C. S.; RASH, G. S.; HONAKER, J. T.; WACHOWIAK, M. P.; SHAW, J. C. A deficient anterior cruciate ligament does not lead to quadriceps avoidance gait. Gait Posture, v.10, n. 3, p. 189-199, 1999.

SHELBURNE, K. B.; TORRY, M. R.; PANDY, M. G. Effect of muscle compensation on knee instability during ACL-deficient gait.Med Sci Sports Exerc., v. 37, n. 4, p. 642-648, 2005.

WEXlER, G.; HURWITZ, D. E.; BUSH-JOSEPH, C. A.; ANDRIACCHI, T. P.; BACH, B. R. 
Functional gait adaptations in patients with anterior cruciate ligament deficiency over time. ClinOrthop., v. 348, p. 166-175, 1998.

ZHANG, L.; SHIAVI, R. G.; LIMBIRD, T. J.; MINORIK, J. M. Six degrees-of-freedom kinematics of ACL deficient knees during locomotion - compensatory mechanism. Gait and Posture, v. 17, p. 34-42, feb. 2003. 